\title{
Understanding the serrated flow and Johari-Goldstein relaxation of metallic glasses
}

\author{
M. Zhang, Y.J. Wang, L.H. Dai* \\ State Key Laboratory of Nonlinear Mechanics, Institute of Mechanics, Chinese Academy of Sciences, Beijing 100190, China
}

\section{A R T I C L E I N F O}

\section{Article history:}

Received 2 March 2016

Received in revised form 19 April 2016

Accepted 20 April 2016

Available online $\mathrm{xxx}$

\section{Keywords:}

Metallic glasses

Nanoindentation

Plastic deformation

Johari-Goldstein relaxation

\begin{abstract}
A B S T R A C T
An increasing tendency of serrated flow with increasing loading rate of a La-based (La) metallic glass (MG) with pronounced Johari-Goldstein relaxation in nanoindentation is investigated, which is abnormal compared to precedent observations where the serrated flow normally decays with increasing loading rate as discovered in the referenced Zr-based (Zr) and Pd-based (Pd) MGs. Based on systematic analysis, a unified dimensionless number is proposed to elucidate the different origins of the abnormal serrated flow of the La MG and the normal serrated flow of the Zr and Pd MGs. The abnormal serrated flow behavior of the La MG provides critical implications in understanding the role of Johari-Goldstein relaxation in the flow of metallic glasses.
\end{abstract}

(c) 2016 Elsevier B.V. All rights reserved.

\section{Introduction}

The origin of the plasticity of metallic glasses [1] (MGs) has been explored with great efforts by studying the rich deformation phenomena of metallic glasses under various circumstances, from monolithic to compound [2], from static to dynamic [3], and from macroscopic to microscopic [4]. Shear band [5] consisting of self-organized shear transformation zones (STZs) [6,7] dominates the plastic deformation of metallic glasses, except in creep [8] or in the deformation of nanometer-sized samples [4]. Due to the "prone-to-failure" character of shear bands, global plasticity of metallic glasses is available only under constrained deformation modes, such as indentation [9], compression [10], and bend [11] etc. In these cases, a distinct trait of the load-displacement curve is the "zigzag" periodical discontinuity of plastic deformation in time domain, i.e. intermittently "burst-out" plastic events, termed serration or serrated flow [12]. Therefore, the mechanism of serrated flow is of great importance to understanding the origin of the plasticity of metallic glasses.

Serrated flow has been widely observed in a variety of metallic glass systems both in physical experiments [13] and in atomistic simulations [14]. The observed serration is proposed to correspond to either the activation of an embryonic shear band or the elementary propagation event of a mature shear band [15]. The statistical character of the size of serrations is related to the nonlinear activation dynamics of the elementary plasticity events in the form of chaos state or self-organized critical state which suggests different ductility of metallic glasses [16]. The stick-slip behavior is also adopted to characterize the serrated

\footnotetext{
* Corresponding author.

E-mail address: lhdai@Inm.imech.ac.cn (L.H. Dai).
}

flow of metallic glasses based on the liquid-like to solid-like transition inside shear bands [17]. In reference to the Portevin-Le Châtelier (PLC) effect in crystal alloys, the serration in the negative strain rate sensitivity regime is explained similar to the dynamic strain aging effect in the deformation of solid solutions [18]. Regardless of the above different explanations, a widely accepted fact is that the serrated flow is strain rate dependent as high rates would usually reduce the intensity of serration [19].

To evaluate the shear band dominated serrated flow of metallic glasses, nanoindentation [20] provides an effective method for its extraordinary high resolution in space on nanometer-sized scale which coincides with the thickness of shear bands of metallic glasses [21]. Moreover, the "constrained" deformation mode in indentation enables versatile and flexible examination on the plastic deformation of metallic glasses in the avoidance of shear banding fracture. On the load $(P)$ displacement $(h)$ curve in nanoindentation tests, the serrated flow of metallic glasses often takes a staircase-like form and presents as "pop-in" events where the displacement exhibits a "forward-jump" at an almost constant load [19]. Normally, the size of the "pop-in" events in nanoindentation would decrease with increasing loading rate at room temperature [22]. Nevertheless, in this work, we report an abnormal, i.e., loading rate-enhanced, serrated flow of a La-based (La) MG [23] with pronounced Johari-Goldstein relaxation [24] and provide a self-consistent explanation to this unusual phenomenon by in-situ examining the creep behavior of metallic glasses under the indenter with the displacement-time curve during the load-hold stage of the indentation program. A unified dimensionless number is proposed to elucidate the different origins of the abnormal serrated flow of the La MG and the normal serrated flow which decreases with increasing loading rates. More importantly, whilst that the Johari-Goldstein relaxation 
has been related to the activation of STZ in the flow of metallic glasses [25], its nature, especially the underlying atomic dynamics involved, remains subtle and elusive [26]. The current work on the abnormal serrated flow of La MG with pronounced Johari-Goldstein relaxation provides meaningful information for identifying the role of Johari-Goldstein relaxation in the flow of metallic glasses.

\section{Experimental}

Alloy sheets of a size of $2 \mathrm{~mm} \times 30 \mathrm{~mm} \times 40 \mathrm{~mm}$ are prepared by copper mould casting under Ti-gettered $\mathrm{Ar}$ atmosphere from master alloys of the typical nominal composition: $\mathrm{Zr}\left(\mathrm{Zr}_{41.2} \mathrm{Ti}_{13.8} \mathrm{Cu}_{12.5} \mathrm{Ni}_{10} \mathrm{Be}_{22.5}\right.$, Vit1), $\mathrm{La}\left(\mathrm{La}_{62} \mathrm{Al}_{14} \mathrm{Ag}_{2.34} \mathrm{Ni}_{10.83} \mathrm{Co}_{10.83}\right)$, and $\mathrm{Pd}\left(\mathrm{Pd}_{40} \mathrm{Cu}_{30} \mathrm{Ni}_{10} \mathrm{P}_{20}\right)$. The amorphous structure of the cast alloy sheets is examined by $\mathrm{X}$-ray Diffraction (XRD) and differential scanning calorimeter (DSC). The samples for nanoindentation were polished to mirror finish before experiments. Nanoindentation tests are conducted on an MTS Nano Indenter XРТ with a Berkovich diamond tip. Load control mode and a group of loading rates $\dot{P}: 0.33 \mathrm{mN} / \mathrm{s}, 1.32 \mathrm{mN} / \mathrm{s}, 13.2 \mathrm{mN} / \mathrm{s}$, and $70 \mathrm{mN} / \mathrm{s}$ are selected. The indentation program is composed by the following stages: (1) load to a maximum force $P_{\max }$ of $200 \mathrm{mN}$ at a constant $\dot{P}$; (2) maintain the load for $10 \mathrm{~s}$ at the maximum force $P_{\max }$; (3) unload to $10 \%$ of the peak load at a rate of $10 \mathrm{mN} / \mathrm{s}$; (4) hold the load for $10 \mathrm{~s}$ to perform thermal drift calibration; (5) unload completely. To guarantee the reliability of the load $(P)$-displacement $(h)$ curve, each test is repeated 7 times.

\section{Results}

Fig. 1 shows the $P$ - $h$ curves of the $\mathrm{La}\left(\mathrm{La}_{62} \mathrm{Al}_{14} \mathrm{Ag}_{2.34} \mathrm{Ni}_{10.83} \mathrm{Co}_{10.83}\right), \mathrm{Zr}$ $\left(\mathrm{Zr}_{41.2} \mathrm{Ti}_{13.8} \mathrm{Cu}_{12.5} \mathrm{Ni}_{10} \mathrm{Be}_{22.5}\right.$, Vit1), and $\mathrm{Pd}\left(\mathrm{Pd}_{40} \mathrm{Cu}_{30} \mathrm{Ni}_{10} \mathrm{P}_{20}\right)$ MGs in nanoindentation. For a clear view, the curves are shifted along the transverse coordinates. The $P$ - $h$ curve obeys the power law equation [27]: $P=C h^{m}$, where $C$ and $m$ are fitting constants depending on loading rate and the shape of indenter. By fitting the $P$ - $h$ curves with the power law equation, it is found that $m$ is about $1.8 \sim 2$ for all the 3 MGs, regardless of the different indentation depths and loading rates, implying similar flow behavior of the $3 \mathrm{MGs}$ on the continuum scale. In Fig. 1, it can be seen that prominent "pop-in" events can be found on the P-h curves of Pd MG (Fig. 1c). However, compared to that of Pd MG, the Zr MG (Fig. 1b) shows slight serrations and the La MG (Fig. 1a) shows no obvious "pop-in" events in the selected loading rate range of the present work.

For a better view on the serrations [28], the $P$ - $h$ curves are subtracted by their fitted curves with the power law equation: $P=C h^{m}$. As shown in Fig. 2, the $\Delta \mathrm{h}-P$ curve, i.e. $\Delta \mathrm{h}$ at the same load $P$, is the difference between each $P$ - $h$ curve and its power law fit. Different serrations can be discerned on the subtracted $P$ - $h$ curves of all the 3 MGs at different loading rate $\dot{P}$. Interestingly, it can be seen in Fig. 2a that with increasing loading rate $\dot{P}$, distinct serrations with a lower frequency than that of the $\mathrm{Zr}$ (Fig. 2b) and Pd (Fig. 2c) MGs emerge on the subtracted $P$ - $h$ curves of La MG. This is abnormal in contrast to the serrations of the $\mathrm{Zr}$ and Pd MGs which decreases with increasing loading rate $\dot{P}$. Similar abnormal serrations have also been found in other $\mathrm{LaAl}(\mathrm{Cu}, \mathrm{Ni}) \mathrm{MGs}$ [28] and Ce-based MGs [29] but at lower loading rates around $0.03 \mathrm{mN} / \mathrm{s}$, where a low glass transition temperature $\left(T_{g}\right)$ is the main cause of the abnormal serrated flow. In previous works, it is proposed that the low $T_{g}$ makes the room temperature $(298 \mathrm{~K}$ ) a relatively high reduced temperature $\left(0.7 \sim 0.8 T_{g}\right)$ at which the viscous flow of metallic glasses is possible at loading rates below $0.03 \mathrm{mN} / \mathrm{s}$. The abnormal serrated flow phenomenon is attributed to $\dot{P}$ induced transition from viscous flow to shear band dominated deformation. However, for the La MG in this work, the $T_{g}$ is $450 \mathrm{~K}$, which gives a reduced value of $0.67 T_{g}$ for room temperature at which the nanoindentation tests are conducted. This value is below the critical value of $0.7 T_{g}$ proposed in the literature $[9,28]$ where locates a crossover from decreasing serrated flow
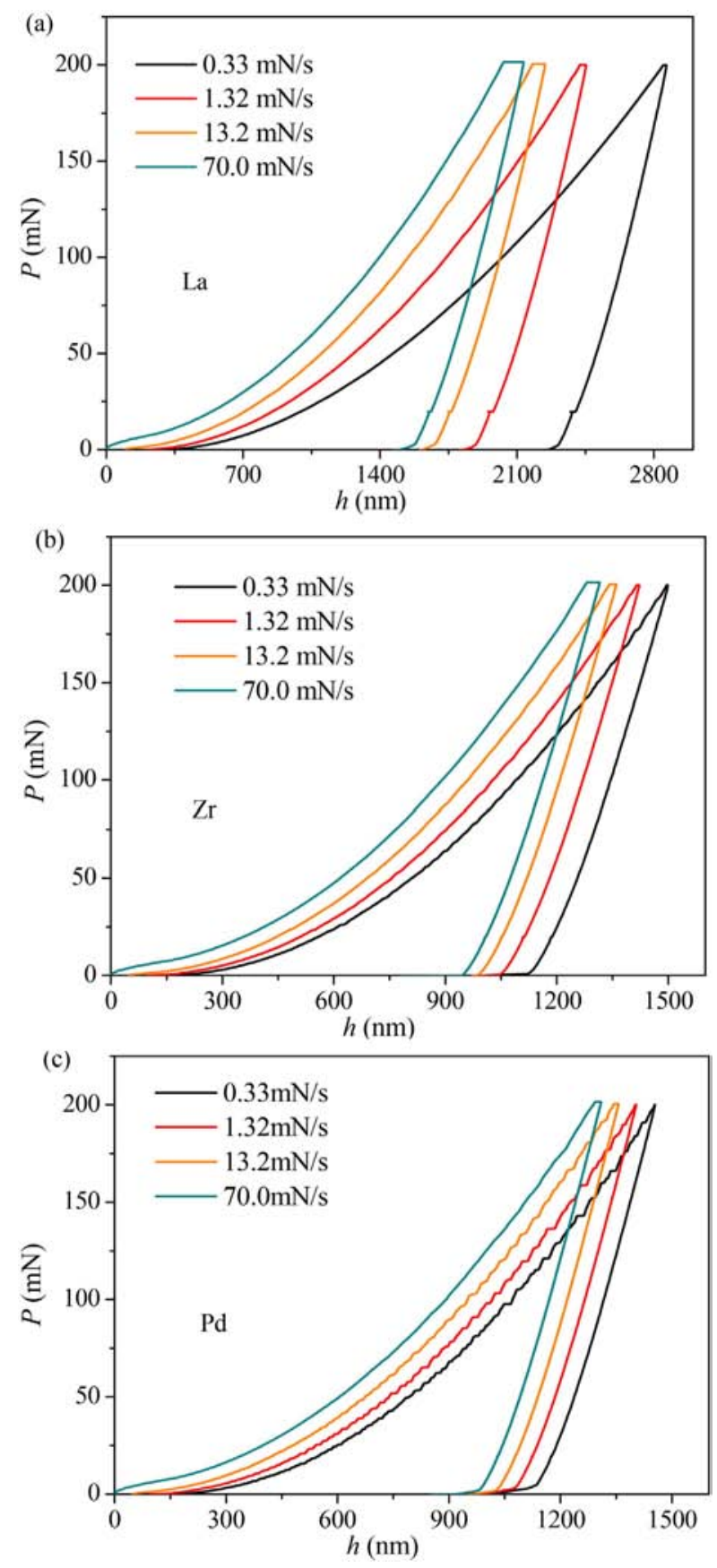

Fig. 1. (Color online) Load-displacement $P$ - $h$ curves of $\mathrm{La}(\mathrm{a}), \mathrm{Zr}$ (b), and $\mathrm{Pd}$ (c) metallic glasses. The curves are shifted along the transverse coordinates.

with increasing $\dot{P}$ to increasing serrated flow with increasing $\dot{P}$. More importantly, according to the flow regimes of metallic glasses [9], for the relatively high loading rates adopted here, the transition from viscous flow to shear band dominated deformation might not be the dominant reason for the abnormal serrated flow of the La MG in Fig. 2a.

To pursue the dominant reason, the in-situ deformation state of metallic glasses at different loading rates under the indenter would be highly useful. Fortunately, the load-hold stage of the indentation program actually functions as a "deformation frozen" technique and captures the in-situ creep deformation behavior of metallic glasses under the indenter [30]. The load-held period of 10s is also in favor of the elimination of the deviations of the indentation depth caused by thermal drift which would grow pronouncedly with extended holding time 

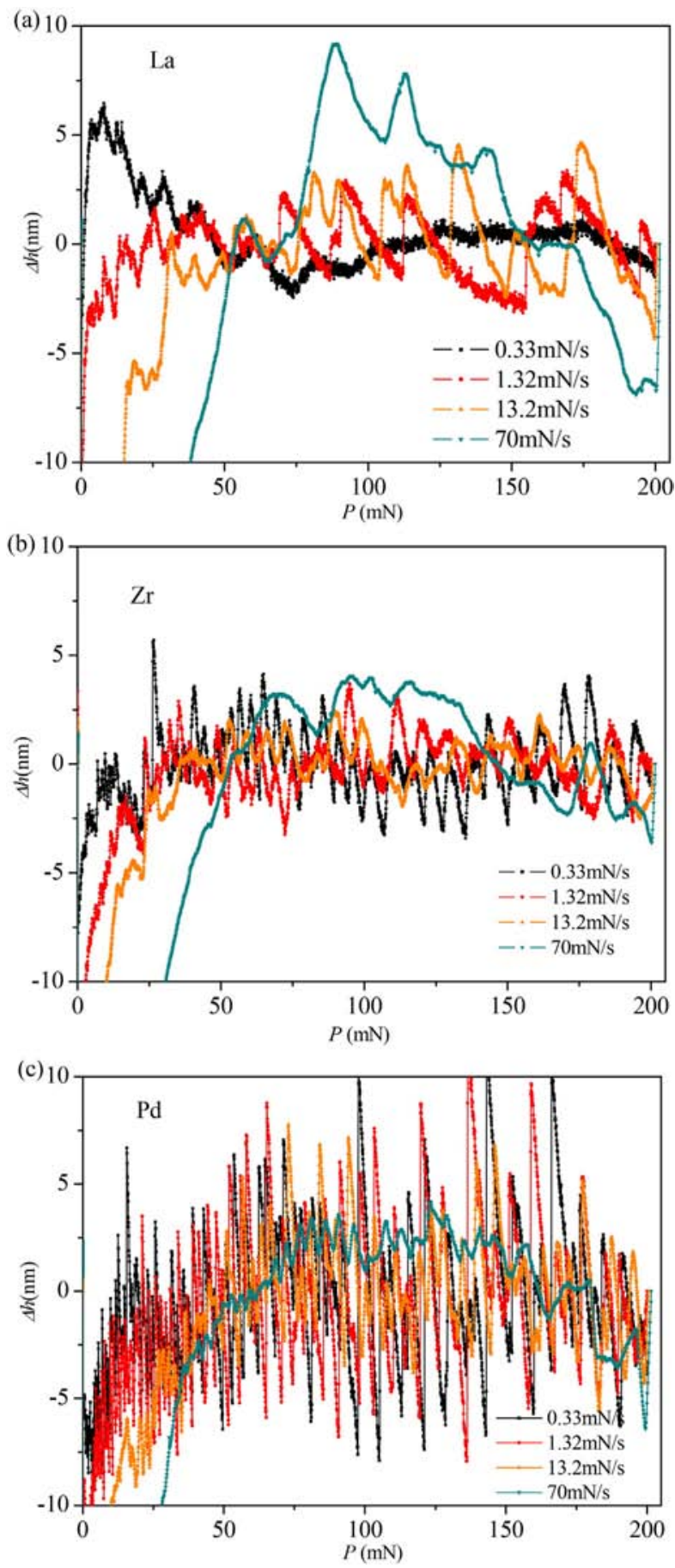

Fig. 2. (Color online) Serrations of $\mathrm{La}(\mathrm{a}), \mathrm{Zr}$ (b), and $\mathrm{Pd}(\mathrm{c}$ ) metallic glasses during the load stage of nanoindentation obtained by subtracting each $P$ - $h$ curve with its power law fit. $\Delta \mathrm{h}$ is the difference between each $P$ - $h$ curve and its power law fit at the same load $P$.

[31]. The displacement-time curves of La, Zr, and Pd MGs during the load-hold stage of the indentation program at different loading rate $\dot{P}$ are shown in Fig. 3. It can be seen that the total creep deformation increases with increasing $\dot{P}$ and that the deformation of all the 3 MGs is approximately saturated after the load held stage suggesting that a period of 10 s for monitoring the creep is appropriate. However, with increasing $\dot{P}$, the time required for the saturation of the creep deformation of $\mathrm{Zr}$ (Fig. 3b) and Pd (Fig. 3c) MGs decreases clearly, but for La MG (Fig. 3a), it is not easy to judge. In the creep flow of metallic glasses, to evaluate the creep rate and the characteristic of the creep process, the
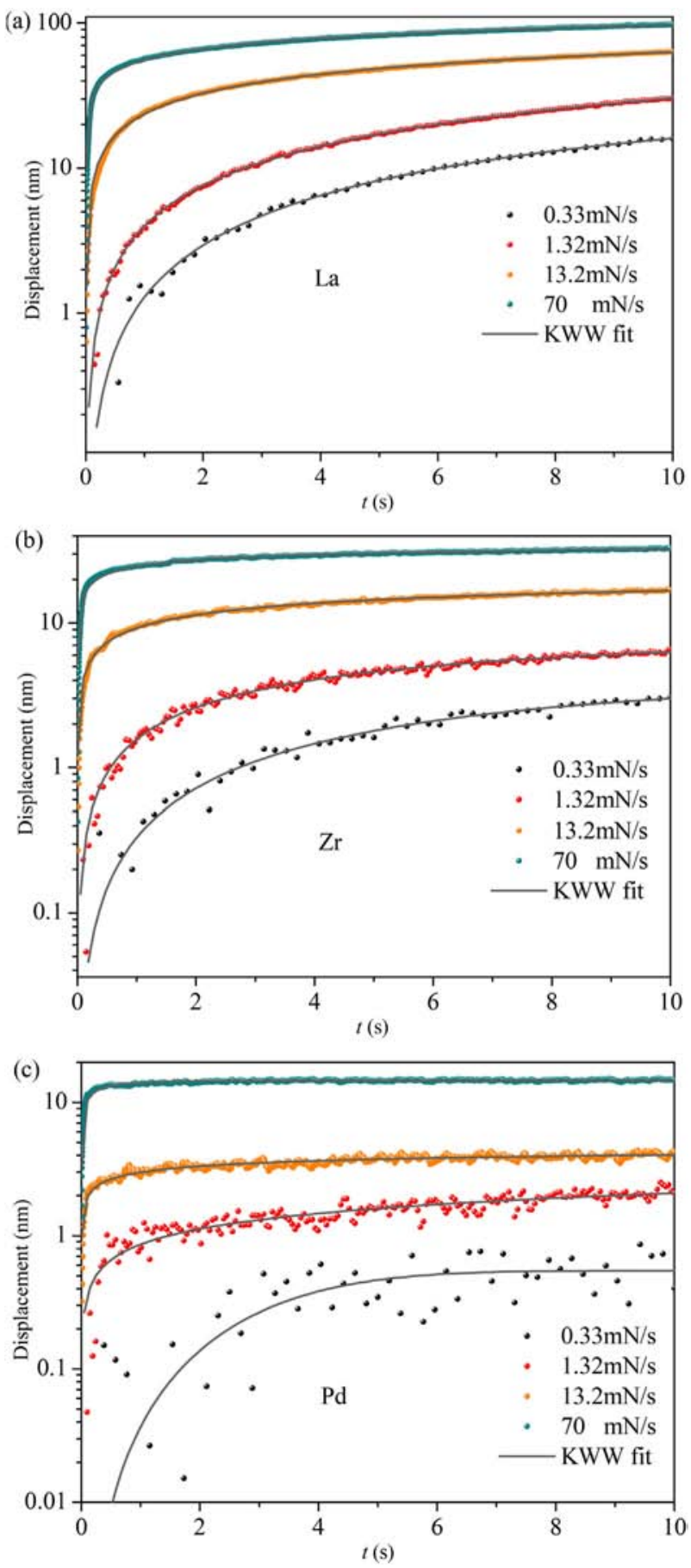

Fig. 3. (Color online) Displacement-time curves of La (a), Zr (b), and Pd (c) metallic glasses of the creep flow during the load-hold stage of nanoindentation. Solid lines are the KWW(Kohlrausch-Williams-Watts) function fits.

time dependent displacement could be empirically approximated with the stretched exponential function: Kohlrausch-Williams-Watts (KWW) [32] equation: $h_{c}=h_{0}\left(1-\exp \left(-\left(t / \tau_{c}\right)^{\beta}\right)\right)$, where $h_{c}$ is the time dependent creep displacement; $h_{0}$ is the total creep displacement, $\tau_{c}$ is the average creep relaxation time, and $\beta$ is the stretched exponent representing the deviation of the creep behavior from the exponential process. Fig. 4 shows the stretched exponent $\beta$ and the average creep relaxation time $\tau_{c}$ of the $3 \mathrm{MGs}$. It can be seen in Fig. 4a that the stretched exponents $\beta$ of all the $3 \mathrm{MGs}$ decrease monotonously and similarly from around 1.5 to 0.5 , indicating the similar broadening distribution of the 
(a)
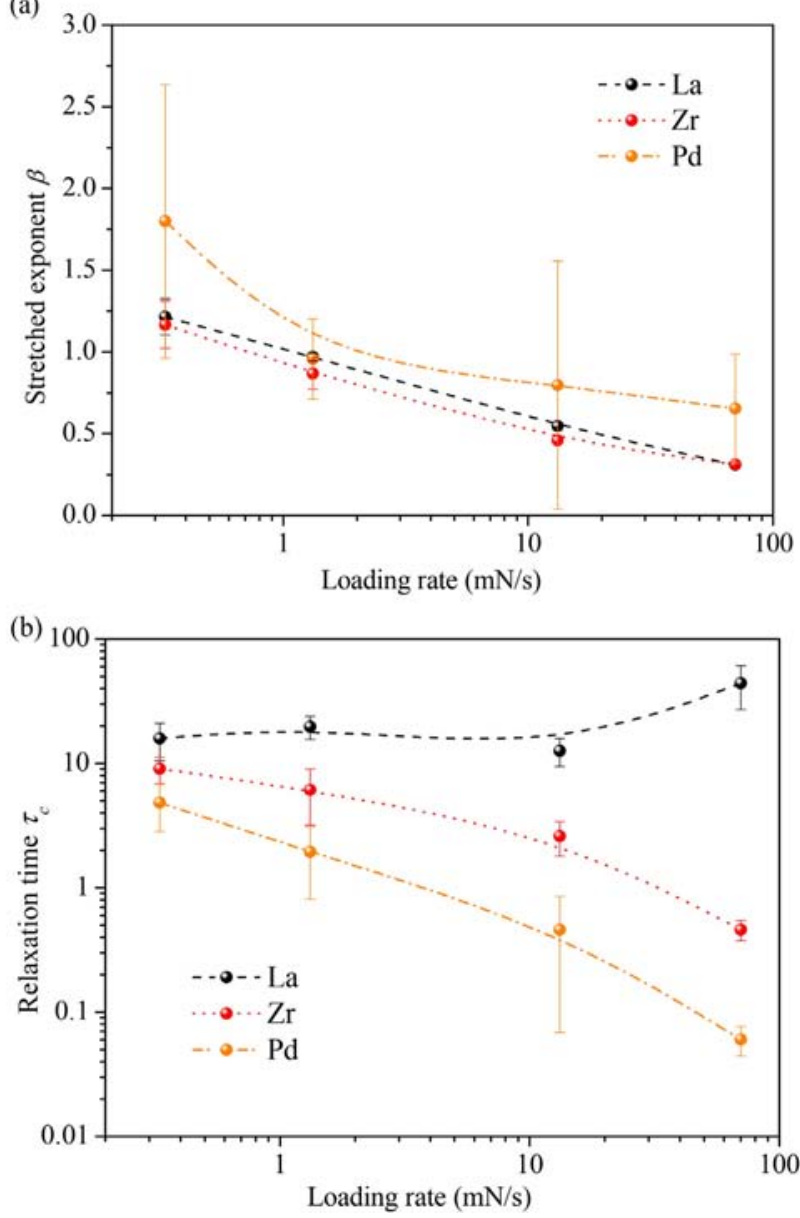

Fig. 4. (Color online) Stretched exponent $\beta(a)$ and average creep relaxation time $\tau_{c}(\mathrm{~b})$ of $\mathrm{La}, \mathrm{Zr}$, and Pd metallic glasses fitted from the displacement-time curves of the creep flow.

activation energy of the STZs in the 3 MGs with increasing $\dot{P}$. The stretched exponent $\beta$ above 1 is proposed to be due to the effect of internal stress [33], which accelerates the creep deformation process. In Fig. $4 \mathrm{~b}$, the average creep relaxation time $\tau_{c}$ of $\mathrm{Zr}$ and Pd MGs decreases similarly from around 10 s to around $0.1 \mathrm{~s}$ as $\dot{P}$ increases from 0.33 to $70 \mathrm{mN} / \mathrm{s}$. In contrast, the average creep relaxation time $\tau_{c}$ of La MG shows an almost constant value of about 20s and barely changes at different loading rate $\dot{P}$, except for a slight increase at $\dot{P}=70 \mathrm{mN} / \mathrm{s}$. It can be seen that the variation of $\tau_{c}$ corresponds well to the serrated flow behavior of the $3 \mathrm{MGs}$ as the decreasing $\tau_{c}$ is observed for $\mathrm{Zr}$ and Pd MGs which exhibit normal serrated flow and the almost constant $\tau_{c}$ is observed for La MG which exhibits abnormal serrated flow. Therefore, it is inferred that the average creep relaxation time $\tau_{c}$ would promisingly be the key to understanding the abnormal serrated flow of La MG. The following concern is to illustrate the possible role played by $\tau_{c}$ in the deformation mechanism of metallic glasses and its relationship with the serrated flow. In the present study, we would like to focus on the mechanical property of the as-fabricated MGs. But, it is noted that the residual stress in the fabricated MGs after the rapid cooling process would have a significant effect on the creep behavior under the indenter. However, according to previous works [30], annealing treatments of increasing aging times which is meant to resolve the residual stress would decrease the total creep deformation but barely affects the characteristic creep relaxation time. Thus, to concentrate on the current issues, it is reasonable that we leave the effect of residual stress on the creep of the 3 MGs for our future works.

\section{Discussion}

\subsection{Origin of the normal serrated flow}

The plastic deformation of metallic glasses is afforded by shear bands [5] consisting of self-organized shear transformation zones (STZs). Based on the operation characteristic of STZs, the elementary strain $\varepsilon_{\text {unit }}$ carried by an STZ is estimated to be $4 \gamma_{c}=0.08$ ( $\gamma_{c}$ is the universal yield strain of metallic glasses) [34]. The incubation and operation of shear bands are perceived as the collective activation of STZs. It is nontrivial to rigorously determine the true operation rate of shear bands. Nevertheless, based on mean field assumptions, the characteristic time in the flow of metallic glasses introduced by strain rate $\dot{\varepsilon}$ can be written in a phenomenological form: $t_{s}=1 / \dot{\varepsilon}$. With increasing $\dot{\varepsilon}$, accelerated shear band operations, i.e., the organization and collective activation of STZs, are demanded. However, the organization and activation of STZs [35] in shear band is determined by the diffusion and percolation of free volume, namely, the time scale of free volume diffusion $\tau_{D}$ would restrict the operation of shear bands [36]. Thus, at high $\dot{\varepsilon}$, multiple shear bands would be cooperatively provoked to meet the requirement of the strain rate and the characteristic time $t_{s}$ can be taken as a measure of the scale of the cooperatively operation of shear bands: high strain rate $\dot{\varepsilon}$ lead to small $t_{s}$ and a large quantity of shear bands, and vice versa. Since serrations correspond to the operation of shear bands, cooperative multiple shear bands would lead to the overlap of large amounts of serrations and smear out the jerky feature on the load-displacement curve. This is the reason why high strain rate $\dot{\varepsilon}$ would suppress the present of serrated flow. These arguments are supported by the network like shear bands pattern underneath the indenter [37] and in metallic glasses deformed under dynamic load [38].

On the other hand, the serrated flow represents the periodically arrested dynamics of shear bands. Therefore, the serrated flow would be highly relevant to the shear band arrest mechanism in metallic glasses. The deformation concentrated in the shear bands would induce a liquid-like state [39], which has been confirmed as the structural disordering [40]. It has been suggested that the arrest of an operating shear band is due to the fact that during serrated flow shear-band propagation occurs in a temperature and strain rate-dependent regime [41] where the structural freezing of the liquid-like state within the shear band (i.e., a liquid-like state to solid-like state transition) is defined by a small characteristic relaxation time $\tau_{r}$ relative to the characteristic time $t_{s}$ of the cooperatively operation of shear bands. It means that, at lower $\dot{\varepsilon}, \tau_{r}$ is sufficiently small compared to $t_{s}$ to quickly reverse the structural softening induced at shear-band initiation, leading to the shear band arrest and thus the serrated flow. On the contrary, at high $\dot{\varepsilon}$ , the stressed shear band remains in a propagating state since $\tau_{r}$ is beyond the characteristic time $t_{s}$ of the cooperatively operation of shear bands where thermally driven atomic rearrangement processes do not allow for structural freezing, and is prone to non-serrated flow.

It can be seen that both the characteristic time $t_{s}$ of cooperatively operation of shear bands and the characteristic relaxation time $\tau_{r}$ of structural freezing inside shear bands are closely related to the normal serrated flow of metallic glasses shown in Fig. 2b-c. However, the abnormal serrated flow shown in Fig. 2a which increases with strain rate cannot be explained based on the deformation mechanism proposed above.

\subsection{Origin of the abnormal serrated flow}

Following the above protocols, as assumed in Part 3, to unravel the origin of the abnormal serrated flow could probably be to unravel the role played by the average creep relaxation time $\tau_{c}$ in the flow of metallic glasses. To do this, it is noted that, as implied in the stick-slip model [42], the serrated flow can also be considered as the intermittent release of anelastic strain into plastic strain along with the collective operation of STZs inside shear bands. The atomistic mechanism of the release of 
anelastic strain can be illustrated as shown in Fig. 5. Microscopically, anelastic strain acting as "friction" in the stick-slip mode operation of STZs is stored in the non-affine displacement (Fig. 5a) of the atoms in an STZ [43]. During the operation of an STZ, upon yielding and as the "Eshelby backstress" is overcome [44], anelastic strain is released into plastic strain in the STZ via atomic rearrangements (see Fig. 5b) signaling a local stress drop (see Fig. 5c). Therefore, macroscopically, the collective operation of large amounts of STZs in the shear band would add up to macroscopic serrated flow. In the mean field sense, the role of the release rate of the anelastic strain into plastic strain during the flow of metallic glasses can be summarized as: i) corresponding to low strain rates, sufficiently rapid release of the anelastic strain which matches the strain rate $\dot{\varepsilon}$ would lead to non-serrated flow; ii) otherwise, corresponding to high strain rates, slow release of anelastic strain which cannot match the strain rate $\dot{\varepsilon}$ would lead to the accumulation of anelastic strain during flow, in which case, to a critical point, as described in the stick-slip model, the accumulated anelastic strain would exhibit a sudden release and lead to serrated flow. Noting that the average creep relaxation time $\tau_{c}$ captures the in-situ anelastic deformation behavior of the metallic glass under the indenter, from this aspect of view, we may interpret the average creep relaxation time $\tau_{c}$ which measures the anelastic flow rate of metallic glasses as an macroscopic empirical release rate of the anelastic strain stored in STZs, as shown in Fig. 5d where the KWW function is equivalent to a tandem of a series of Kelvin models of different relaxation times $\tau_{1}, \tau_{2}, \cdots$, and $\tau_{\infty}$ which represents the distribution of the STZs of different activation energy barrier. From a different angle, the abnormal serrated flow due to the slow release of anelastic strain can be understood in analogue to the stress overshoot [45] phenomenon usually observed in the flow of supercooled liquids which increases rapidly with strain rates. As pointed out in literatures [44], the stress overshoot represents the transition of the reversible local atomic rearrangements to irreversible global configuration reconstruction in the flow of metallic glass, which is exactly the transition procedure of the anelastic strain into plastic strain depicted above.

Hence, it is obvious that the serrated flow of metallic glasses depends on the relation between the characteristic time of cooperatively operation of shear bands $t_{s}$ and the average creep relaxation time $\tau_{c}$. Moreover, the release rate of anelastic strain also depends on the diffusion rearrangement of atoms, i.e., the viscosity, in the shear band. Therefore, the characteristic relaxation time $\tau_{r}$ of the structural freezing in the arrest of shear bands is intimately related to the average creep relaxation time $\tau_{c}$ [41]. A longer $\tau_{r}$ for structural freezing inside shear bands represents a lower viscosity state in the shear band, and in turn a shorter average creep relaxation time $\tau_{c}$ and a larger creep displacement $h_{0}[41]$.

\subsection{Application to La, Zr, and Pd metallic glasses}

Now, the different serrated flow behaviors of the 3 MGs can be interpreted as follows. As shown in Fig. 4b, the average creep relaxation times $\tau_{c}$ for $\mathrm{Zr}$ and Pd MGs decrease significantly as loading rate $\dot{P}$ increasing from $0.33 \mathrm{mN} / \mathrm{s}$ to $70 \mathrm{mN} / \mathrm{s}$, indicating an increasing characteristic relaxation time $\tau_{r}$. In these cases, as shown in Fig. 2b-c, the serrated flow of $\mathrm{Zr}$ and Pd MGs decreases with $\dot{P}$ for both the increasing $\tau_{r}$ which delays the structural freezing leading to less serrated flow [41] and the decreasing $\tau_{c}$ which speeds the release of the anelastic strain leading to less serrated flow. However, for La MG, the average creep relaxation time $\tau_{c}$ remains almost constant with increasing $\dot{P}$, indicating an almost constant characteristic relaxation time $\tau_{r}$. Although the increasing $\dot{P}$ would suppress the formation of serrated low for the decreasing characteristic time $t_{s}$ of cooperatively operation of multiple shear bands and for the almost constant characteristic relaxation time $\tau_{r}$ at different loading rates which relatively delays the structural freezing comparing to the increased loading rate $\dot{P}$, as argued previously [28], the increasing $\dot{P}$ will also result into a relatively lower release rate of the anelastic strain comparing to the increased loading rate $\dot{P}$ due to the almost constant average creep relaxation time $\tau_{c}$ at different loading rates and would lead to serrated flow. This explains why La MG exhibits enhanced serrated flow with increasing $\dot{P}$.

On the other hand, as stated in previous section, the serrated flow due to the slow release of anelastic strain can also be understood in analogue to the stress overshoot. It is also widely reported that the stressstrain curve which exhibits stronger stress overshoot also exhibits a steeper elastic stage, suggesting a higher modulus and more elastic energy stored in the supercooled metallic liquids [45]. Fig. 6 shows the ratio $W_{U} / W_{L}$ of unload work $W_{U}$ to load work $W_{L}$ [46] calculated based on the loading curves and unloading curves of the $3 \mathrm{MGs}$ respectively. This ratio indicates the elastic energy stored in metallic glasses in the loading process. A larger value of $W_{U} / W_{L}$ indicates more work is released during the unloading stage, i.e. more work is stored as elastic (a)

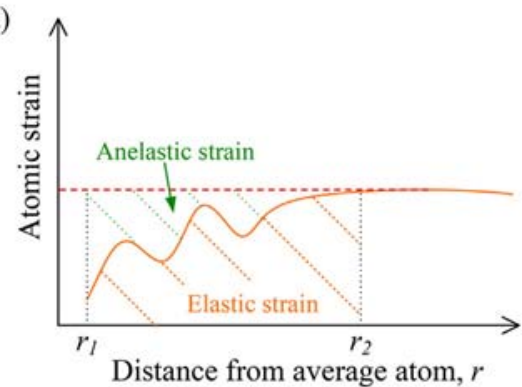

(c)

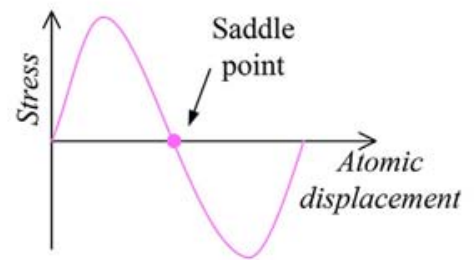

(b)

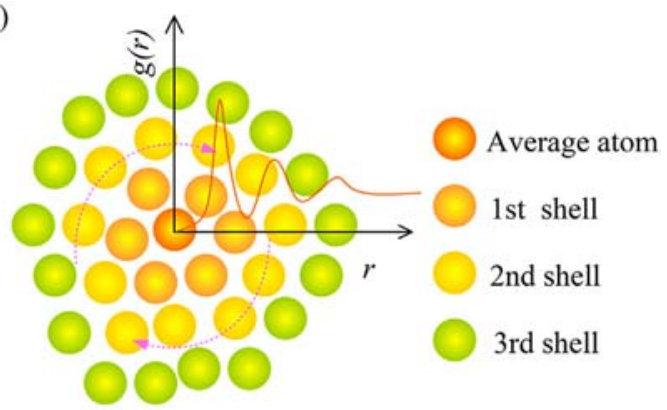

(d)

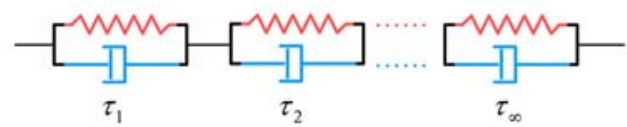

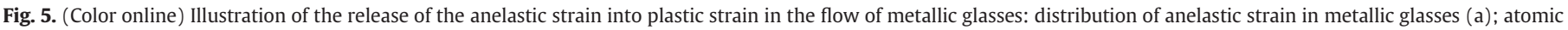

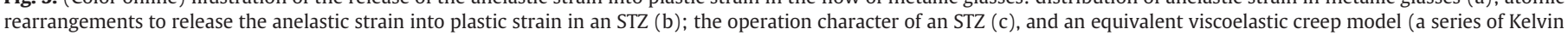
models of relaxation time $\tau_{1}, \tau_{2}, \cdots$, and $\tau_{\infty}$ representing the distribution of STZs) for the KWW (Kohlrausch-Williams-Watts) function(d). 


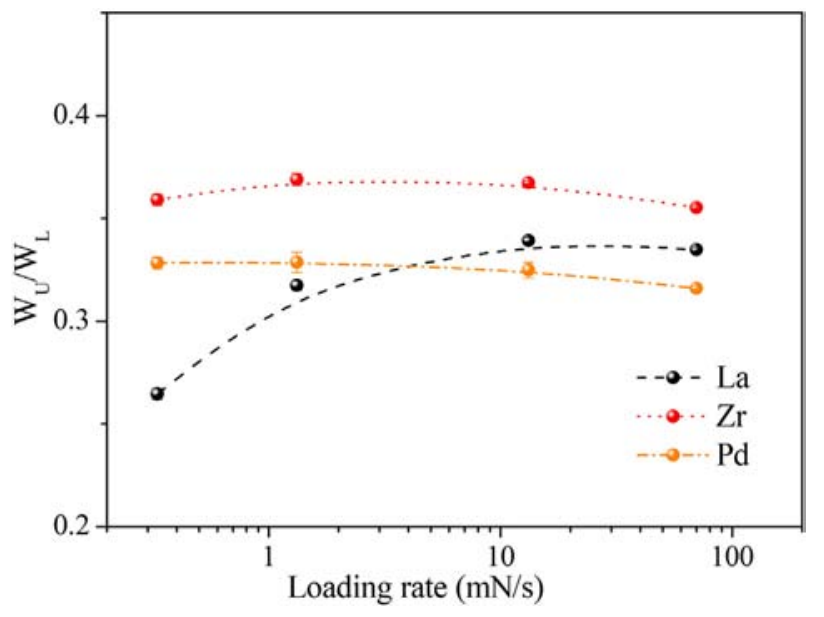

Fig. 6. (Color online) The ratio of unload work to load work of $\mathrm{La}, \mathrm{Zr}$, and Pd metallic glasses.

energy during the loading stage. It can be seen that the ratio $W_{U} / W_{L}$ increases for La MG and barely changes for $\mathrm{Zr}$ or Pd MG with increasing loading rate, indicating that larger fraction of load work is stored as elastic energy in La MG at higher loading rates, whereas the faction of load work stored as elastic energy in $\mathrm{Zr}$ and Pd MGs does not change much with increasing loading rate. Therefore, the result of Fig. 6 where more elastic energy is stored at higher loading rates is consistent with the deductions above that the origin of the abnormal serrated flow of La MG is due to its low release rate of anelastic strain. What's more, based on the shear banding instability index proposed based on the stiffness of the sample and the frame of the test machine [47], more elastic energy stored in the sample reflects the increase of the stiffness of the sample with increasing loading rate, i.e., a relatively decreased stiffness of the frame of the test machine. The relatively higher stiffness of the sample would enhance the instable nature of shear banding and lead to more serrated flow. From this aspect, the result of Fig. 6 again supports our conclusion that the increasing tendency of serrated flow with increasing loading rate in La MG is due to its low release rate of anelastic strain into plastic strain which increases the sample stiffness and the elastic energy stored in metallic glasses.

\subsection{A unified dimensionless number}

Before proceeding, some remarks should be clearly made here. For the complexity introduced by the correlated evolution dynamics of STZ and free volume in the deformation of metallic glasses [7,35,48], it would seem too ambitious to try to distinctly separate the different origins of the serrated flow of metallic glasses. Taking the complicated stress states and the deformation modes in nanoindentation into account, also due to the rather simplified concept of the characteristic times based on mean field assumptions proposed above and the diverse plasticity of different metallic glasses, it is also rational to concede that a rigorously accurate and universal criterion for the serrated flow of metallic glasses is beyond the capability of quantitative and analytic characterization. However, it is still achievable that the different origins of the serrated flow of different metallic glasses can be understood with a clear presentation, because that as discussed above, the serrated flow of metallic glasses can be rationalized with the characteristic times involved in deformation.

Totally, the main characteristic times in the shear band-dominated serrated flow of metallic glasses can be summarized as follows:

(1) $t_{s}=1 / \dot{\varepsilon}$ is the characteristic time of the external deformation rate and characterizes the cooperatively operation rate of shear bands, in nanodentation [49], $\dot{\varepsilon}=\dot{P} / 2 P_{\max }$;
(2) $\tau_{e}=\sqrt{\rho A^{2} / G}$ is the characteristic time [50] of elastic deformation and characterizes the elastic response rate inside an STZ. $\rho$ is the density of metallic glasses; $A$ is the diameter of STZ; $G$ is shear modulus.

(3) $\tau_{c}$ is the average creep relaxation time representing the release rate of anelastic displacement of atoms and can be understood as the internal time scale of the matter transport in the flow which is intimately related to the process of the JohariGoldstein relaxation; $\tau_{c}$ can also be estimated from the nominal viscous flow time $\eta_{\text {nom }} / E_{r}$ of the $3 \mathrm{MGs}$ in nanoindentation, where $\eta_{\text {nom }}=\sigma / 3 \dot{\varepsilon}=H / 9 \dot{\varepsilon} ; \sigma$ is flow stress [49]; $H$ is the hardness and $E_{r}$ is the reduced modulus determined in nanoindentation.

(4) $\tau_{r}$ is the characteristic relaxation time of structural freezing [41] and characterize the annihilation rate of free volume inside shear bands, which determines the normal serrated flow originating from the arrest or "frozen" of an operating shear band.

With these characteristic times, based on previous work [50] a unified dimensionless number: $I_{\text {serration }}=\tau_{e} / t_{s}+\tau_{e} / \tau_{c}+\tau_{c} / t_{s}+t_{s} / \tau_{r}$ can be established to characterize the serrated flow in metallic glasses. The first 3 terms on the right hand side phenomenologically indicates the deformation evolution inside shear bands and the 4th term phenomenologically indicates the structure evolution inside shear bands. Since $\tau_{e}=\sqrt{\rho A^{2} / G}$ is of an order of $10^{-12} \mathrm{~s}$ indicating the instantresponse assumption of elasticity, the first 2 terms would be crucial in dynamic tests. But for the loading rates adopted here, $t_{s} \sim\left(10^{0}, 10^{3} \mathrm{~s}\right)$ and $\tau_{c} \sim\left(10^{-1}, 10^{1} \mathrm{~s}\right)$, the first 2 terms can be approximated as 0 . The 3rd term and the 4 th term are left in the dimensionless number: $I_{\text {serration }}=\tau_{c} / t_{s}+t_{s} / \tau_{r}$ and the boundary condition for the serrated flow of metallic glasses can be written as $I_{\text {serration }} \geq I_{c}$, where the critical constant $I_{c}$ depends on the composition of metallic glasses. The different origins of the serrated flow can hereby be classified as follows. At low strain rate $\left(\dot{\varepsilon}<\dot{\varepsilon}_{c} \propto 1 / \tau_{c}\right)$, i.e., $t_{s}=1 / \dot{\varepsilon}$ is large, the 3 rd term $\tau_{c} / t_{s}$ approximates 0 . The 4 th term $t_{s} / \tau_{r}$ dominates and leads to normal serrated flow which decreases with increasing strain rate. At high strain rate $\left(\dot{\varepsilon}>\dot{\varepsilon}_{c} \propto 1 / \tau_{c}\right)$, i.e., $t_{s}=1 / \dot{\varepsilon}$ is small, the 4 th term $t_{s} / \tau_{r}$ approximates 0 . The 3rd term $\tau_{c} / t_{s}$ dominates and leads to abnormal serrated flow which increases with increasing strain rate. As show in Fig. 4b, the former case is for $\mathrm{Zr}$ and Pd MGs, which show a small $\tau_{c}$ corresponding to a high critical strain rate $\dot{\varepsilon}_{c-Z r}$ or $\dot{\varepsilon}_{c-P d}$. The latter case is for La MG which shows a large $\tau_{c}$ corresponding to a low critical strain rate $\dot{\varepsilon}_{c-L a}$. Thus, the dimensionless number $I_{\text {serration }}$ indicates that , within the range of the loading rate adopted in this work, the $\mathrm{Zr}$ and Pd MGs deform in the low strain rate regime $\left(\dot{\varepsilon}_{\varepsilon} \dot{\varepsilon}_{c-Z r}\right.$ and $\left.\dot{\varepsilon}<\dot{\varepsilon}_{c-P d}\right)$ and show the 4 th term $t_{s} / \tau_{r}$ dominated serrated flow which normally decreases with increasing loading rate; the La MG deforms in the high strain rate regime $\left(\dot{\varepsilon}>\dot{\varepsilon}_{c-L a}\right)$ shows the 3 rd term $\tau_{c} / t_{s}$ dominated serrated flow which abnormally increases with increasing loading rate. The fact that La MG deforms in the high strain rate regime $\left(\dot{\varepsilon}>\dot{\varepsilon}_{c-L a}\right)$ corroborates well our postulation in Fig. 2, where the reason for the abnormal serration of La MG is proposed to be not due to the $\dot{P}$ induced transition from viscous flow to shear band dominated deformation at low strain rate around $0.03 \mathrm{mN} / \mathrm{s}$ for the low glass transition temperature of La MG.

An important manifestation for the different origins of the serrated flow of La and $\mathrm{Zr}$ (or Pd) MGs is the different serration frequency between La and Pd( or Zr) MGs, as shown in Fig. 2, where the low frequency serration of La MG indicates the low release rate of the anelastic deformation $\tau_{c}$ and slow atomic rearrangements inside the shear bands, whereas the high frequency serration of $\mathrm{Pd}$ (or Zr) MG reflects the short characteristic relaxation time $\tau_{r}$ and the fast free volume evolution dynamics inside shear bands. The fast free volume evolution dynamics relative to the slow atomic rearrangements induced by deformation inside shear bands would possibly explain why the shear 
band thickness determined by the variation of nanoindentation hardness is much larger [51] than the shear band thickness observed under transmission electron microscopy [52], because the expansion of the deformation boundary of shear band lays behind the free volume diffusion.

\subsection{Understanding the Johari-Goldstein relaxation}

Hence, the above results indicate that in addition to the characteristic time $t_{\mathrm{s}}$ of the external deformation and the characteristic relaxation time $\tau_{r}$ of structural freezing inside shear bands, the characteristic time $\tau_{c}$ of the release of anelastic strain in STZs is another key factor in the serrated flow of metallic glasses. Based on the intimate correlation between the operation of STZ and Johari-Goldstein relaxation [25], the almost constant average creep relaxation time $\tau_{c}$, i.e., the constant release rate of the anelastic strain in STZ, of La MG is probably due to its pronounced Johari-Goldstein relaxation at room temperature [23] in contrast to $\mathrm{Zr}$ and Pd MGs (See also Supplementary Material), which effectively cancels the shear dilation in the localized deformation process of STZs leading to an almost constant release rate of the anelastic deformation at different loading rates $\dot{P}$ or in another word: an apparently Newtonian-like viscous creep behavior with a constant relaxation time.

To justify the propositions above, Fig. 7 shows the nominal viscous flow time $\eta_{\text {nom }} / E_{r}$ of the $3 \mathrm{MGs}$ in nanoindentation under different loading rates. Theoretically, the nominal viscous flow time $\eta_{\text {nom }} / E_{r}$ should be equivalent to the average creep relaxation time $\tau_{c}$. The average creep relaxation time $\tau_{c}$ is also shown here for comparison. It can be seen that the nominal viscous flow time of the 3 MGs decrease monotonously with increasing loading rate because of shear dilation inside shear bands. For Pd and Zr MGs, the nominal viscous flow time $\eta_{\text {nom }} / E_{r}$ indeed effectively characterizes the average creep relaxation time $\tau_{c}$ indicated by the similar order of magnitude of their values. However, for La MG, although exhibiting a decreasing nominal viscous flow time $\eta_{\text {nom }} / E_{r}$ like $\mathrm{Zr}$ and $\mathrm{Pd} \mathrm{MGs}$, an almost constant average creep relaxation time $\tau_{c}$ is exhibited to indicate the outrageous anelastic behavior of La MG during nanoindentation under the selected loading rates in this study. For a better understanding on the nominal viscous flow time $\eta_{\text {nom }} / E_{r}$, the $3 \mathrm{rd}$ term $\tau_{c} / t_{s}$ of the dimensionless number $I_{\text {serration }}$ is adopted here and written into $\frac{\eta_{\text {nom }} / E_{r}}{t_{s}}$, by substituting $\eta_{\text {nom }} / E_{r}$ for $\tau_{c}$. As shown in Fig. 8, it can be seen that the dimensionless number $\frac{\eta_{\text {nom }} / E_{r}}{t_{s}}$ increases for La MG and barely changes for Zr and Pd MGs with increasing loading rate. Because that the dimensionless number $\frac{\eta_{\text {nom }} / E_{r}}{t_{s}}$ characterizes the competition between the internal viscous flow of metallic glass with

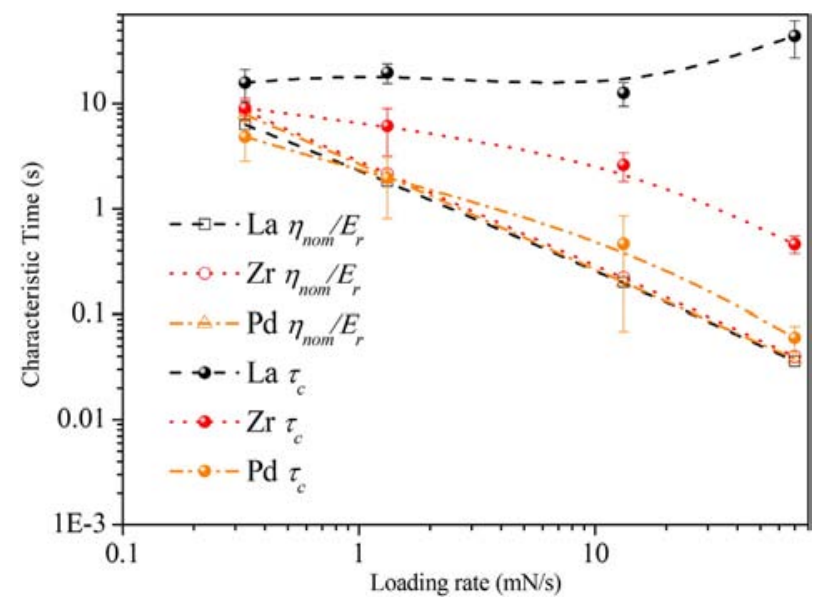

Fig. 7. (Color online) The nominal viscous flow time $\eta_{\text {nom }} / E_{r}$ of La, $\mathrm{Zr}$, and Pd metallic glasses.

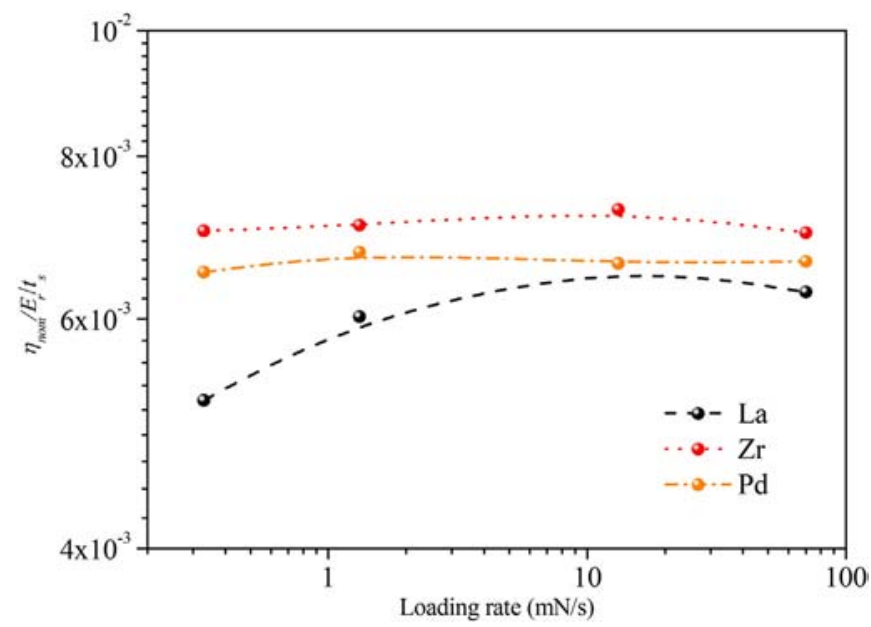

Fig. 8. (Color online) The dimensionless number $\frac{\eta_{\text {nom }} / E_{T}}{t_{s}^{s}}$ of La, Zr, and Pd metallic glasses.

the external loading rate, it correlates well with the ratio $W_{U} / W_{L}$ of unload work $W_{U}$ to load work $W_{L}$. Therefore, it is inferred that the nominal viscous flow time $\eta_{\text {nom }} / E_{r}$ reflects the macroscopic flow of metallic glasses and the average creep relaxation time $\tau_{c}$ probably reflects the microscopic and local flow character of metallic glasses. Due to the role of STZ in the flow mechanism of metallic glasses as localized hidden flow [53], this discrepancy between the nominal viscous flow time $\eta_{\text {nom }} /$ $E_{r}$ and the average creep relaxation time $\tau_{c}$ of La MG could be a convincing proof for the proposition that the pronounced Johari-Goldstein relaxation of La MG which mainly determines the local flow of metallic glasses is the main reason for the almost constant average creep relaxation time $\tau_{c}$. In this sense, the above results would provide a new angle to understand the nature of the Johari-Goldstein relaxation in metallic glasses which might be localized Newtonian viscous flow in the shear transformation zones. Remembering the breakdown of the EinsteinStokes equation accompanying the supercooling of the glass forming liquids [54], the almost constant average creep relaxation time $\tau_{c}$ would also be enlightening to understanding the peculiar diffusion property of metallic glasses.

Another issue to be noted is that like the pioneering works [30], our results on the creep deformation of metallic glasses also indicate that the loading stage would significantly affect the creep rate and creep characteristics of metallic glasses. Similar effects of the loading stage can be conceived in the stress relaxation tests of metallic glasses. Therefore, more attentions should be paid to this issue to accurately understand the mechanical properties of metallic glasses such as: creep, stress relaxation, anelasticity, and so on.

\section{Conclusions}

To summarize, abnormal serrated flow which increases with loading rate of a La MG with pronounced Johari-Goldstein relaxation in nanoindentation is observed in contrast to the normal serrated flow of $\mathrm{Zr}$ and Pd MGs where the serration decays with increasing loading rate. The abnormal serrated flow of La MG is interpreted in the frame work of the stick-slip model with its almost constant average creep relaxation time at different loading rates obtained by fitting the displacementtime curve during the load-hold stage of the indentation program with the KWW (Kohlrausch-Williams-Watts) function. The different origins of the normal and abnormal serrated flow of metallic glasses are elucidated with a unified dimensionless number. The almost constant average creep relaxation time of La MG suggests that the nature of the Johari-Goldstein relaxation in metallic glasses could be localized Newtonian viscous flow in the shear transformation zones. 


\section{Acknowledgement}

This work was financially supported by the National Nature and Science foundation of China under grants No. 11472287 and No. 11402269 , the National Basic Research Program of China under grants No. 2012CB937500, and the CAS/SAFEA International Partnership Program for Creative Research Teams.

\section{Appendix A. Supplementary data}

Supplementary data to this article can be found online at http://dx. doi.org/10.1016/j.jnoncrysol.2016.04.036.

\section{References}

[1] C.A. Schuh, T. Hufnagel, U. Ramamurty, Mechanical behavior of amorphous alloys, Acta Mater. 55 (2007) 4067-4109.

[2] Y.C. Choi, S.I. Hong, Enhancement of plasticity in Zr-base bulk metallic glass by soft metal plating, Scr. Mater. 61 (2009) 481-484.

[3] L.F. Liu, L.H. Dai, Y.L. Bai, B.C. Wei, Initiation and propagation of shear bands in $\mathrm{Zr}$ based bulk metallic glass under quasi-static and dynamic shear loadings, J. NonCryst. Solids 351 (2005) 3259-3270.

[4] H. Guo, P.F. Yan, Y.B. Wang, J. Tan, Z.F. Zhang, M.L. Sui, E. Ma, Tensile ductility and necking of metallic glass, Nat. Mater. 6 (2007) 735-739.

[5] A.L. Greer, Y.Q. Cheng, E. Ma, Shear bands in metallic glasses, Mater. Sci. Eng. R. Rep. 74 (2013) 71-132.

[6] M.L. Falk, J.S. Langer, Dynamics of viscoplastic deformation in amorphous solids, Phys. Rev. E 57 (1998) 7192-7205.

[7] E.R. Homer, C.A. Schuh, Mesoscale modeling of amorphous metals by shear transformation zone dynamics, Acta Mater. 57 (2009) 2823-2833.

[8] H.B. Ke, P. Wen, H.L. Peng, W.H. Wang, A.L. Greer, Homogeneous deformation of metallic glass at room temperature reveals large dilatation, Scr. Mater. 64 (2011) 966-969.

[9] C.A. Schuh, A.C. Lund, T.G. Nieh, New regime of homogeneous flow in the deformation map of metallic glasses: elevated temperature nanoindentation experiments and mechanistic modeling, Acta Mater. 52 (2004) 5879-5891.

[10] Y.H. Liu, G. Wang, R.J. Wang, Q. Zhao de, M.X. Pan, W.H. Wang, Super plastic bulk metallic glasses at room temperature, Science 315 (2007) 1385-1388.

[11] Y. Chen, M.Q. Jiang, L.H. Dai, Collective evolution dynamics of multiple shear bands in bulk metallic glasses, Int. J. Plast. 50 (2013) 18-36.

[12] S.X. Song, T.G. Nieh, Direct measurements of shear band propagation in metallic glasses - An overview, Intermetallics 19 (2011) 1968-1977.

[13] A.R. Yavari, J.J. Lewandowski, J. Eckert, Mechanical properties of bulk metallic glasses, MRS Bull. 32 (2007) 635-638.

[14] S.Y. Jiang, M.Q. Jiang, L.H. Dai, Y.G. Yao, Atomistic Origin of Rate-Dependent Serrated Plastic Flow in Metallic Glasses, Nanoscale Res. Lett. 3 (2008) 524-529.

[15] D. Klaumunzer, A. Lazarev, R. Maass, F.H.D. Torre, A. Vinogradov, J.F. Loffler, Probing Shear-Band Initiation in Metallic Glasses, Phys. Rev. Lett. 107 (2011) 185502.

[16] B.A. Sun, H.B. Yu, W. Jiao, H.Y. Bai, D.Q. Zhao, W.H. Wang, Plasticity of Ductile Metallic Glasses: A Self-Organized Critical State, Phys. Rev. Lett. 105 (2010) 035501.

[17] D. Klaumünzer, R. Maaß, J.F. Löffler, Stick-slip dynamics and recent insights into shear banding in metallic glasses, J. Mater. Res. 26 (2011) 1453-1463.

[18] F.H. Dalla Torre, A. Dubach, M.E. Siegrist, J.F. Loffler, Negative strain rate sensitivity in bulk metallic glass and its similarities with the dynamic strain aging effect during deformation, Appl. Phys. Lett. 89 (2006) 091918.

[19] C.A. Schuh, T.G. Nieh, A nanoindentation study of serrated flow in bulk metallic glasses, Acta Mater. 51 (2003) 87-99.

[20] C.A. Schuh, T.G. Nieh, A survey of instrumented indentation studies on metallic glasses, J. Mater. Res. 19 (2004) 46-57.

[21] M.Q. Jiang, W.H. Wang, L.H. Dai, Prediction of shear-band thickness in metallic glasses, Scr. Mater. 60 (2009) 1004-1007.

[22] C.A. Schuh, T.G. Nieh, Y. Kawamura, Rate dependence of serrated flow during nanoindentation of a bulk metallic glass, J. Mater. Res. 17 (2002) 1651-1654.

[23] D.D. Liang, X.D. Wang, Y. Ma, K. Ge, Q.P. Cao, J.Z. Jiang, Decoupling of pronounced beta and alpha relaxations and related mechanical property change, J. Alloys Compd. 577 (2013) 257-260.

[24] G.P. Johari, M. Goldstein, Viscous Liquids and the Glass Transition. II. Secondary Relaxations in Glasses of Rigid Molecules, J. Chem. Phys. 53 (1970) 2372-2388.
[25] H.B. Yu, W.H. Wang, H.Y. Bai, Y. Wu, M.W. Chen, Relating activation of shear transformation zones to beta relaxations in metallic glasses, Phys. Rev. B 81 (2010) 220201

[26] Y.H. Liu, T. Fujita, D.P.B. Aji, M. Matsuura, M.W. Chen, Structural origins of JohariGoldstein relaxation in a metallic glass, Nat. Commun. 5 (2014) 3238.

[27] I.N. Sneddon, The relation between load and penetration in the axisymmetric boussinesq problem for a punch of arbitrary profile, Int. J. Eng. Sci. 3 (1965) 47-57.

[28] W. Peng, B. Wei, T. Zhang, Y. Liu, L. Li, Characterization of shear banding in La-based bulk metallic glasses through indentation, Mater. Trans. 48 (2007) 1759-1764.

[29] B.C. Wei, L.C. Zhang, T.H. Zhang, D.M. Xing, J. Das, J. Eckert, Strain rate dependence of plastic flow in Ce-based bulk metallic glass during nanoindentation, J. Mater. Res. 22 (2007) 258-263.

[30] A. Castellero, B. Moser, D.I. Uhlenhaut, F.H. Dalla Torre, J.F. Loffler, Roomtemperature creep and structural relaxation of $\mathrm{Mg}-\mathrm{Cu}-\mathrm{Y}$ metallic glasses, Acta Mater. 56 (2008) 3777-3785.

[31] V. Maier, B. Merle, M. Goken, K. Durst, An improved long-term nanoindentation creep testing approach for studying the local deformation processes in nanocrystalline metals at room and elevated temperatures, J. Mater. Res. 28 (2013) 1177-1188.

[32] G. Williams, D.C. Watts, Non-symmetrical dielectric relaxation behaviour arising from a simple empirical decay function, Trans. Faraday Soc. 66 (1970) 80-85.

[33] T. Morishita, Compressed exponential relaxation in liquid silicon: Universal feature of the crossover from ballistic to diffusive behavior in single-particle dynamics, J. Chem. Phys. 137 (2012) 024510-024516.

[34] W.L. Johnson, K. Samwer, A Universal Criterion for Plastic Yielding of Metallic Glasses with a $\left(T / T_{g}\right)^{2 / 3}$ Temperature Dependence, Phys. Rev. Lett. 95 (2005) 195501.

[35] L. Li, E.R. Homer, C.A. Schuh, Shear transformation zone dynamics model for metallic glasses incorporating free volume as a state variable, Acta Mater. 61 (2013) 3347-3359.

[36] L.H. Dai, M. Yan, L.F. Liu, Y.L. Bai, Adiabatic shear banding instability in bulk metallic glasses, Appl. Phys. Lett. 87 (2005) 141916.

[37] U. Ramamurty, S. Jana, Y. Kawamura, K. Chattopadhyay, Hardness and plastic deformation in a bulk metallic glass, Acta Mater. 53 (2005) 705-717.

[38] F. Zeng, Y. Chen, M.Q. Jiang, C. Lu, L.H. Dai, Dynamic fragmentation induced by network-like shear bands in a Zr-based bulk metallic glass, Intermetallics 56 (2015) 96-100.

[39] P.F. Guan, M.W. Chen, T. Egami, Stress-Temperature Scaling for Steady-State Flow in Metallic Glasses, Phys. Rev. Lett. 104 (2010) 205701.

[40] P. de Hey, J. Sietsma, A. van den Beukel, Structural disordering in amorphous Pd40Ni40P20 induced by high temperature deformation. Acta Mater. 46 (1998) 5873-5882.

[41] R. Maaß, D. Klaumünzer, G. Villard, P.M. Derlet, J.F. Löffler, Shear-band arrest and stress overshoots during inhomogeneous flow in a metallic glass, Appl. Phys. Lett. 100 (2012) 071904.

[42] F.H. Dalla Torre, D. Klaumunzer, R. Maass, J.F. Loffler, Stick-slip behavior of serrated flow during inhomogeneous deformation of bulk metallic glasses, Acta Mater. 58 (2010) 3742-3750.

[43] M. Zhang, Y.J. Wang, L.H. Dai, Bridging shear transformation zone to the atomic structure of amorphous solids, J. Non-Cryst. Solids 410 (2015) 100-105.

[44] J.S. Harmon, M.D. Demetriou, W.L. Johnson, K. Samwer, Anelastic to plastic transition in metallic glass-forming liquids, Phys. Rev. Lett. 99 (2007) 135502.

[45] M.Q. Jiang, G. Wilde, L.H. Dai, Origin of stress overshoot in amorphous solids, Mech. Mater. 81 (2015) 72-83.

[46] Y.T. Cheng, C.M. Cheng, Relationships between hardness, elastic modulus, and the work of indentation, Appl. Phys. Lett. 73 (1998) 614-616.

[47] Z. Han, W.F. Wu, Y. Li, Y.J. Wei, H.J. Gao, An instability index of shear band for plasticity in metallic glasses, Acta Mater. 57 (2009) 1367-1372.

[48] A.S. Argon, Plastic deformation in metallic glasses, Acta Metall. 27 (1979) 47-58.

[49] D. Pan, A. Inoue, T. Sakurai, M.W. Chen, Experimental characterization of shear transformation zones for plastic flow of bulk metallic glasses, Proc. Natl. Acad. Sci. U. S. A. 105 (2008) 14769-14772.

[50] X. Huang, Z. Ling, L.H. Dai, Cavitation instabilities in bulk metallic glasses, Int. J. Solids Struct. 50 (2013) 1364-1372.

[51] J. Pan, Q. Chen, L. Liu, Y. Li, Softening and dilatation in a single shear band, Acta Mater. 59 (2011) 5146-5158

[52] M.W. Chen, A. Inoue, W. Zhang, T. Sakurai, Extraordinary plasticity of ductile bulk metallic glasses, Phys. Rev. Lett. 96 (2006) 245502.

[53] Z. Wang, B.A. Sun, H.Y. Bai, W.H. Wang, Evolution of hidden localized flow during glass-to-liquid transition in metallic glass, Nat. Commun. 5 (2014) 5823.

[54] U. Geyer, S. Schneider, W.L. Johnson, Y. Qiu, T.A. Tombrello, M.P. Macht, Atomic diffusion in the supercooled liquid and glassy states of the zr41.2til3.8cu12.5ni10be22.5 alloy, Phys. Rev. Lett. 75 (1995) 2364-2367. 